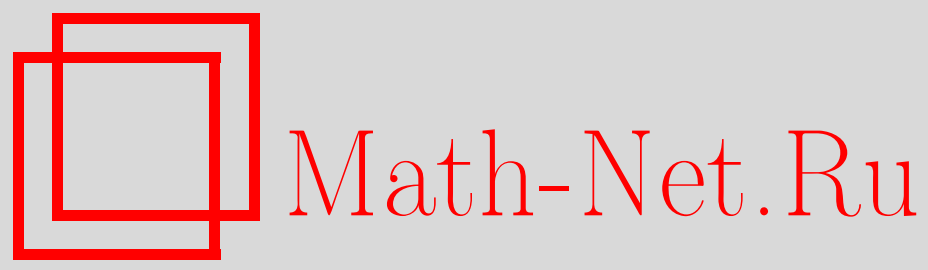

C. В. Асташкин, О мультипликаторе симметричного пространства относительно тензорного произведения, Функи. анализ и его прил., 1996, том 30, выпуск 4, 58-60

DOI: https://doi.org/10.4213/faa550

Использование Общероссийского математического портала MathNet.Ru подразумевает, что вы прочитали и согласны с пользовательским соглашением

http://www . mathnet.ru/rus/agreement

Параметры загрузки:

IP : 54.172 .240 .79

26 апреля 2023 г., 18:37:21

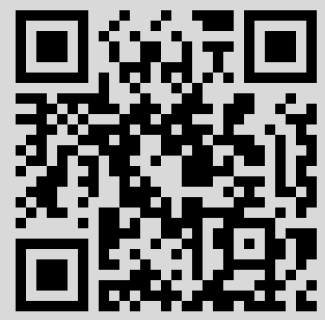




\title{
О мультипликаторе симметричного пространства относительно тензорного произведения
}

\author{
(c) 1996. С. В. АСТАшкин
}

Пусть $x=x(s)$ и $y=y(t)$ 一 измеримые функции на $I=[0,1]$. Определим билинейный оператор

$$
B(x, y)(s, t)=x \otimes y(s, t)=x(s) y(t), \quad(s, t) \in I \times I .
$$

Напомним, что банахово пространство $E$ измеримых функций на $I$ называется симметричным (с.п.), если из того, что $y \in E$ и $x^{*}(t) \leqslant y^{*}(t)$ (здесь $z^{*}(t)$ - убывающая перестановка функции $|z(u)|[1$, с. 93]), следует, что $x \in E$ и $\|x\| \leqslant\|y\|$.

Если $E$ - с.п. на $I$, то соответствующее с.п. на квадрате $E(I \times I)$ состоит из всех измеримых функций $x=x(s, t)$ на $I \times I$, для которых $x^{*} \in E$ и $\|x\|_{E(I \times I)}=\left\|x^{*}\right\|_{E}$.

Если $X, Y, Z-$ с.п. на $I$, то непрерывность оператора $B$ из $X \times Y$ в $Z(I \times I)$ эквивалентна непрерывности вложения проективного тензорного произведения $X \otimes Y$ в $Z$ [2, p. 51]. По этой причине его называют оператором тензорного произведения.

Важность изучения оператора $B$ обусловлена тем, что он естественным образом возникает при решении целого ряда задач, связанных с изучением геометрических свойств с.п. (см., например, [3, p. 169-171; 4, 5]).

Большая часть работ по изучению тензорного произведения в с.п. посвяшена нахождению условий его непрерывности в их конкретных классах: в пространствах Лоренца, Марцинкевича, Орлича и некоторых других $[2,6-9]$. В этой заметке речь пойдет о произвольных с.п. Введем основное для дальнейшего понятие мультипликатора с.п. относительно оператора $B$.

Пусть $E-$ с.п. на $I$. Его мультипликатором назовем множество $M(E)$ всех измеримых на $I$ функций $x=x(s)$, для которых $x \otimes y \in E(I \times I)$ с произвольной функцией $y \in E$. Тогда $M(E)$ линейно, а после введения нормы

$$
\|x\|_{M(E)}=\sup \left\{\|x \otimes y\|_{E(I \times I)} ;\|y\|_{E} \leqslant 1\right\}
$$

становится с.п. на $I$. Очевидно, что всегда $M(E) \subset E$.

Рассмотрим пример. Пусть $E$ - пространство Лоренца $\Lambda(\phi)$, где $\phi(u)-$ неотрицательная возрастающая вогнутая функщия на $(0,1]$. Напомним, что оно состоит из всех измеримых функций $x=x(s)$, для которых

$$
\|x\|_{\Lambda(\phi)}=\int_{0}^{1} x^{*}(s) d \phi(s)<\infty .
$$

Предположим, что для функщии растяжения

$$
\mathscr{M}_{\phi}(v)=\sup \{\phi(t v) / \phi(t), 0<t \leqslant \min (1,1 / v)\}
$$


выполнено условие

$$
\mathscr{M}_{\phi}(v)=\lim _{t \rightarrow t_{0}} \phi(t v) / \phi(t),
$$

где $t_{0} \in[0,1]$ не зависит от $v \in[0,1]$. Тогда несложные вычисления показывают, что $M(\Lambda(\phi))=\Lambda\left(\mathscr{M}_{\phi}\right)$.

Пусть $\chi_{e}(s)=1(s \in e), \chi_{e}(s)=0(s \notin e)$ (e- измеримое подмножество в $I$ или в $I \times I)$. Важную роль в теории с.п. играют фундаментальная функция с.п. $\phi_{E}(t)=\left\|\chi_{(0, t)}\right\|_{E}$, а также непрерывный в любом с.п. оператор растяжения $\sigma_{t} y(u)=y(u / t) \chi_{[0,1]}(u / t)(t>0)$.

Теорема 1. Для любого с.n. E на I

$$
\begin{gathered}
\phi_{M(E)}(t)=\left\|\sigma_{t}\right\|_{E \rightarrow E}, \quad 0<t \leqslant 1, \\
\left\|\sigma_{t}\right\|_{M(E) \rightarrow M(E)}=\left\|\sigma_{t}\right\|_{E \rightarrow E}, \quad 0<t \leqslant 1, \\
\left\|\sigma_{1 / t}\right\|_{E \rightarrow E}^{-1} \leqslant\left\|\sigma_{t}\right\|_{M(E) \rightarrow M(E)} \leqslant\left\|\sigma_{t}\right\|_{E \rightarrow E}, \quad t>1 .
\end{gathered}
$$

Неравенства (2), вообще говоря, нельзя заменить равенством, аналогичным (1). Чтобы это показать, достаточно рассмотреть случай, когда $\phi_{\alpha}(s)=$ $s^{\alpha} \ln ^{-1}(C / s), E=\Lambda\left(\phi_{\alpha}\right)(0<\alpha<1), C>\exp \{1 /(1-\alpha)\}$, и воспользоваться результатом приведенного выше примера.

Напомним, что для любого с.п. $E$ существуют числа

$$
\alpha_{E}=\lim _{t \rightarrow 0} \ln \left\|\sigma_{t}\right\|_{E \rightarrow E} / \ln t, \quad \beta_{E}=\lim _{t \rightarrow \infty} \ln \left\|\sigma_{t}\right\|_{E \rightarrow E} / \ln t,
$$

которые называются нижним и верхним индексами Бойда пространства $E[1]$. Всегда $0 \leqslant \alpha_{E} \leqslant \beta_{E} \leqslant 1$ [1, с. 134].

СЛЕДСТВИЕ 1. Для индексов Бойда с.п. E и $M($ Е) выполнены неравенства $\alpha_{E} \leqslant \alpha_{M(E)} \leqslant \beta_{M(E)} \leqslant \beta_{E}$.

Теорема 2 (оценка сверху). Пусть $E-c . n$. на $I$ и $p=1 / \alpha_{E}$, әде $\alpha_{E}-$ нижний индекс Бойда с.п. E. Тогда $M(E) \subset L_{p}$, и константа вложения не зависит от $E$.

СЛЕДСТВИЕ 2. Если $\alpha_{E}=0$, mo $M(E)=L_{\infty}$.

Если $E$ - с.п. на $I$, то ассоциированное с ним пространство $E^{\prime}$ состоит из всех измеримых на $I$ функций $y=y(t)$, для которых

$$
\|y\|_{E^{\prime}}=\sup \left\{\int_{0}^{1} x(t) y(t) d t ;\|x\|_{E} \leqslant 1\right\}<\infty .
$$

Норма с.п. $E$ называется порядково полунепрерывной, если из монотонной сходимости $x_{n} \uparrow x$ п.в. $\left(x_{n}, x \in E\right)$ следует, что $\left\|x_{n}\right\| \rightarrow\|x\|$.

СЛЕДСТВИЕ 3. Пусть $E$ - c.n. c порядково полунепрерьвной нормой. Если $B: E \times E \rightarrow E(I \times I)$ u $B: E^{\prime} \times E^{\prime} \rightarrow E^{\prime}(I \times I)$, mo $E=L_{p} \partial_{\Omega}$ некоторого $p \in[1, \infty]$.

ТЕОРема 3 (оценка снизу). Дяя љюбого с.n. E на I 
где $\psi(t)=\left\|\sigma_{t}\right\|_{E \rightarrow E}(0<t \leqslant 1)$.

Константа вложения при этом не зависит от $E$.

Перейдем к конкретным результатам. Напомним, что пространство $L_{p q}(1<$ $p<\infty, 1 \leqslant q \leqslant \infty)$ состоит из всех измеримых на $I$ функций $x=x(t)$, для которых эквивалентный норме функционал

$$
\|x\|_{p q}= \begin{cases}\left\{\int_{0}^{1}\left(x^{*}(t) t^{1 / p}\right)^{q} d t / t\right\}^{1 / q}, & \text { если } 1 \leqslant q<\infty, \\ \sup _{0<t \leqslant 1}\left(x^{*}(t) t^{1 / p}\right), & \text { если } q=\infty,\end{cases}
$$

конечен.

Теорема 4. Пусть с.n. E интерполяиионно между $L_{p}$ и $L_{p, \infty}$ при некотором $p \in(1, \infty)$ (т.е. из ограниченности произвольного линейного оператора в $L_{p}$ и $L_{p, \infty}$ следует его непрерьвность в $\left.E\right)$. Тогда $M(E)=L_{p}$.

Так как $L_{p q}(p \leqslant q \leqslant \infty)$ интерполяционно между $L_{p}$ и $L_{p, \infty}[1$, c. 142], получаем

СлЕДСТВИЕ 4. Если $1<p<\infty, p \leqslant q \leqslant \infty$, то $M\left(L_{p q}\right)=L_{p}$.

В случае $q=\infty$ последнее утверждение было доказано в [9].

В заключение приведем одно приложение полученных результатов в связи с известной теоремой Р. О'Нейла о непрерывности оператора тензорного произведения в пространствах $L_{p, q}$.

Теорема 5 [8]. Пусть $1<p<\infty, 1 \leqslant q, r, s \leqslant \infty$. Оператор $B$ является непрерывным оператором из $L_{p r} \times L_{p s}$ в $L_{p q}(I \times I)$, если и только если выполнень условия

(1) $\max (s, r) \leqslant q$;

(2) $1 / p+1 / q \leqslant 1 / s+1 / r$.

Следствие 4 показывает, что в случае $s=p<r=q$ теорема 5 точна относительно класса всех с.п. (а не только пространств $L_{p q}$ ). А именно, $L_{p}-$ максимальное среди с.п. $E$, для которых $B$ является ограниченным оператором из $L_{p r} \times E$ в $L_{p r}(I \times I)$.

\section{ЛиТеРАтУРА}

1. Крейн С. Г., Петунин Ю. И., Семенов Е. М. Интерполяция линейных операторов. Наука, M., 1978. 2. Milman M. Notas Mat., 20, 1-128 (1978). 3. Lindenstrauss J., Tzafriri L. Classical Banach Spaces. Vol. 2. Function Spaces. SpringerVerlag, Berlin, 1979. 4. Johnson W. B., Maurey B., Schechtman G., Tzafriri L. Symmetric Structures in Banach Spaces. Mem. Am. Math. Soc., Vol. 217, 1979, pp. 1-298. 5. Carothers N. L. Israel J. Math., 40, No. 3-4, 217-228 (1981). 6. Milman M. Notas Mat., 13,1-7 (1977). 7. Milman M. Anal. Math., 4, No. 3, 215-223 (1978). 8. O'Neil R. J. d'Analyse Math., 21, 129-142 (1968). 9. Асташкин С. В. В сб.: Исследования по теории функций многих вещественных переменных. Ярославль (1982), с. 3-15. 